\title{
Novel electromagnetic-based navigation for percutaneous transforaminal endoscopic lumbar decompression in patients with lumbar spinal stenosis reduces radiation exposure and enhances surgical efficiency compared to fluoroscopy: a randomized controlled trial
}

\author{
Junlong Wu ${ }^{1,2 \#, ~ S h e n g x i a n g ~ A o ~}{ }^{1 \#}$, Huan Liu ${ }^{1}$, Wenkai Wang ${ }^{1}$, Wenjie Zheng ${ }^{1}$, Changqing Li ${ }^{1}$, \\ Chao Zhang ${ }^{1}$, Yue Zhou ${ }^{1}$ \\ ${ }^{1}$ Department of Orthopaedics, Xinqiao Hospital; Army Medical University, Chongqing, China; ${ }^{2}$ Department of Orthopaedics, the 941 Hospital of \\ Chinese People Liberation Army, Xining, China \\ Contributions: (I) Conception and design: J Wu, Y Zhou; (II) Administrative support: Y Zhou, C Zhang; (III) Provision of study materials or patients: \\ J Wu, S Ao, H Liu, C Li; (IV) Collection and assembly of data: W Wang, W Zheng; (V) Data analysis and interpretation: J Wu, S Ao, Y Zhou; (VI) \\ Manuscript writing: All authors; (VII) Final approval of manuscript: All authors. \\ \#These authors contributed equally to this work. \\ Correspondence to: Yue Zhou; Chao Zhang. Department of Orthopaedics, Xinqiao Hospital, Army Medical University, Xinqiao Main Street 183, \\ Shapingba District, Chongqing, China. Email: happyzhou@vip.163.com; tmmuzc@163.com.
}

\begin{abstract}
Background: Percutaneous transforaminal endoscopic lumbar decompression (PTELD) is an emerging surgical alternative for treating lumbar spinal stenosis (LSS). However, the foraminoplasty procedure often requires repeated fluoroscopy, and endoscopy just offers a local view. No studies have focused on decreasing radiation exposure with electromagnetic navigation assistance. This study introduces a novel electromagnetic-based navigation (EMN) endoscopic system for PTELD in patients with LSS and compares the results in navigation and fluoroscopy groups.

Methods: Eighty-eight patients with LSS were randomized into either a navigation (44 patients) or fluoroscopy group. Duration of surgery, cannula placement time, radiation dose, blood loss, intraoperative pain assessment, and postoperative hospitalization stay were evaluated. The clinical outcomes were evaluated using a visual analogue scale (VAS), the Oswestry Disability Index (ODI), 6-minute walk test, and modified Macnab criteria.

Results: Eighty-five patients were followed-up for at least 12 months. The duration of surgery and cannula placement time were significantly more efficient in the navigation group $(\mathrm{P}=0.03$ and $\mathrm{P}<0.001)$. Intraoperative pain assessment showed significantly less pain in the navigation group $(\mathrm{P}=0.038)$. The radiation dose was significantly higher in the fluoroscopy group than the navigation group $(\mathrm{P}<0.001)$. The VAS scores for back $(\mathrm{P}<0.001)$ and leg $(\mathrm{P}<0.001)$ pain improved significantly in both groups after surgery, as did the ODI $(\mathrm{P}<0.001)$ scores. Improvements in walking ability and Macnab criteria assessments at the 12-month follow-up, assessed subjective by patient assessments did not differ between the two groups.

Conclusions: The EMN system used in PTELD for patients with LSS compared to fluoroscopy enhances efficiency for foraminoplasty, reduces intraoperative pain and levels of radiation exposure. It results in outcomes comparable with results using fluoroscopy.
\end{abstract}

Keywords: Endoscopy; electromagnetic navigation assistance; fluoroscopy; lumbar spinal stenosis (LSS); percutaneous transforaminal endoscopic lumbar decompression (PTELD)

Submitted Feb 24, 2020. Accepted for publication Aug 19, 2020.

doi: $10.21037 /$ atm-20-1877

View this article at: http://dx.doi.org/10.21037/atm-20-1877 


\section{Introduction}

Lumbar spinal stenosis (LSS) is characterized by narrowing of the spinal canal as a consequence of bone and soft tissue degeneration, including osteophyte formation, degeneration of the ligamentum flavum, disc herniation, and facet hypertrophy (1-4).

Traditionally, LSS is treated with open laminectomy, foraminotomy, or fusion $(5,6)$. Recently, a minimally invasive spine (MIS) surgical technique was developed to improve preservation of the surrounding normal anatomical structures and to diminish intraoperative bleeding, thus reducing recovery times $(7,8)$. Microscopic bilateral decompression via a unilateral approach has been effectively demonstrated in the treatment of LSS (9). With development of the percutaneous endoscopic technique, percutaneous endoscopic decompression via the transforaminal approach is also an emerging surgical alternative for treating LSS $(10,11)$. The procedure can be performed with local anesthesia, especially in older patients with concurrent, serious, or medical illnesses who cannot tolerate conventional surgery under general anesthesia (12). However, the partial field of vision is restricted, and technical difficulties can arise despite the use of spinal endoscope, even for experienced endoscopic surgeons (13). Furthermore, the traditional percutaneous endoscopic lumbar discectomy technique has a flat learning curve (14). The design and intraoperative application of the proper trajectory for puncture in foraminoplasty for LSS are highly demanding of experience and technique (15). Cases with a high iliac crest or severe migration magnify the puncture difficulty (16). The extremely minimal access of the percutaneous endoscopic technique necessitates precise fluoroscopic localization of anatomic landmarks to allow for preoperative determination of optimal starting points and trajectories. Intraoperative exposure to radiation is an indispensable part of the percutaneous endoscopic technique, and minimization of exposure is a major concern for both patients and the surgical team.

In this article, we introduce a novel electromagneticbased navigation (EMN) endoscopic system (SEEssys, Joimax-China, Fiagon, Germany) for percutaneous transforaminal endoscopic lumbar decompression (PTELD) in patients with LSS. We hypothesized that the novel endoscopic EMN technique would result in higher efficiency in foraminoplasty and lower levels of radiation exposure compared to imaging-guided fluoroscopy. We analyzed the results between navigation and fluoroscopy groups in a prospectively controlled case series. We present the following article in accordance with the CONSORT reporting checklist (available at http://dx.doi.org/10.21037/ atm-20-1877).

\section{Methods}

According to a pre-defined protocol, between April 2018 and October 2018, following approval from the Ethics Committee of The Army Medical University (written informed consent was obtained from all patients), Eightyeight patients from the teaching hospital of a medical university in southwestern China agreed to participate. The study was conducted in accordance with the Declaration of Helsinki (as revised in 2013). The work has been reported in line with the CONSORT 2010 Statement (17). All the patients were assigned randomly by a computergenerated randomization process, in a $1: 1$ ratio, to the navigation group or the fluoroscopy group. For allocation concealment, the randomization allocation results of each patient were deposited in a light-proof envelope by the statistician and were not revealed to the surgeon until the operation day. This random controlled clinical trial was registered in a public trial registry (ChiCTR1900028523).

The inclusion criteria were: age is between 18 and 80 years; diagnosis is single-level LSS combined with disc herniation (DH); the main complain symptoms is unilateral neurological intermittent claudication or radicular leg pain refractory to nonsurgical management for at least 12 weeks; and absence of significant instability. The exclusion criteria were: symptoms without herniation or sciatica; severe osseous central stenosis; inoperable medical disease; peripheral nerve disease; instability; multilevel LSS; previous lumbar surgery; spinal infections; ossification of the ligamentum flavum; traumatic lesions; and presence of bony metastasis. The diagnosis of LSS was based on the presence of typical symptoms and morphology of the dural sac on the cross-section on MRI $(4,5)$.

Perioperative data, including duration of surgery, cannula placement time, radiation dose, blood loss, and intraoperative pain assessment, as well as postoperative hospitalization stay, were recorded. Intraoperative pain was assessed as follows: "none" = mild or no pain from the puncture, "moderate" = moderate pain from the puncture for a short time, "severe" = severe pain from the puncture for a long time, and "intolerable" = pain of such a degree that the operation should be stopped (15).

The primary outcome was the score on the Oswestry 
Disability Index (ODI, which ranges from 0 to 100). Second clinical outcomes were evaluated using a visual analogue scale (VAS, which range from 0 to 10), and modified Macnab criteria (18). A 3-point improvement in the VAS score and a 12-point improvement in the ODI score were considered the minimum clinically important difference (19). All clinical follow-up variables were collected preoperatively and postoperatively at 1-, 3-, 6-, and 12-month follow-up by two clinic researchers who were not involved in the operations. In addition to the patient reported outcome measures, the 6-minute walk test (the distance that a patient can walk in 6 minutes) was assessed by a trial nurse at baseline and at the 12-month follow-up (20).

In the navigation group, two patients were excluded due to the plan bias in the operation. The mean follow-up was $14.5 \pm 2.2$ months. During the follow-up period, one patient in the navigation group was lost to follow-up, leaving 41 navigation (group A) patients and 44 non-navigation (group B) patients for final analysis.

\section{Surgical technique}

All surgeries were performed by one experienced endoscopic surgeon.

\section{Navigation group}

The patient was placed in a prone position on a special, nonmetallic, carbon fiber OR table to prevent electromagnetic interference. The field generator was placed on the gluteal or ventral side of the patient so that the electromagnetic field encompassed the entire surgical field. The K-wire was drilled or driven $2 \mathrm{~cm}$ deep into the spinous process of the vertebral body, which was the nearest caudal vertebra among the relevant vertebral bodies. The localizer was fixed to the $\mathrm{K}$-wire at a distance $5-10 \mathrm{~mm}$ from the skin. Then, the patient tracker was attached to the spinous process to form the reference for the coordination system. The mapper bridge, which was placed just next to the localizer, was identified by at least 17 markers in the anteroposterior and lateral images for a high possibility of successful registration (Figure 1). The mapper bridge was positioned on the back so that all the mapper symbols were green. After lateral and anteroposterior images were taken, the mapper bridge was not moved. Then, the fluoroscopy images were transferred to the navigation system via a USB drive. Once data were loaded, the system automatically performed registration. The system could not correct static errors that were attributable to the position and alignment of the navigation instrument. After confirming registration, intraoperative $2 \mathrm{D}$ images were used to match the preoperative CT data. Then, navigation was carried out virtually, in real time, in a $3 \mathrm{D}$ data set.

The entire surgery was performed under local anesthesia and optional narcotic sedation. The stylet was removed from the $18 \mathrm{G}$ needle, and the IseePointer sensor was inserted into the needle and locked with a Luer connection. The needle was held on the multipad until the needle symbol appeared in the top right corner. A total of $15-30 \mathrm{~mL}$ of $0.5 \%$ lidocaine was infiltrated into the puncture trajectory through the needle. Under the guidance of real-time inline navigation views, the optimal foraminoplasty trajectory on the superior articular process (SAP) for intracanal exposure and neurological decompression based on different clinical diagnoses could be easily designed and obtained (Figure 2). A puncture guidance angle was also given during puncture, and green was displayed when the angle was correct. Then, the following surgical procedure was performed: (I) a guide wire was passed through the needle, and the needle was removed; (II) a skin incision (approximately $1.5 \mathrm{~cm}$ ) was made at the entry point of the guide wire; (III) the guiding rod was held on the multipad until the symbol was chosen automatically; (IV) the guiding rod was inserted along the guide wire with the inline views toward the SAP; (V) a halfserrated working tube was inserted with the navigation rod, which consisted of the IseePointer and adapter, and was then removed; (VI) an appropriate reamer with a handle was introduced along the half-serrated working cannula; (VII) the endoscope with the Iseepointer in the left flushing canal was inserted; (VIII) the ventral osteophyte on the SAP could be resected safely under the inline navigation views and was removed by forceps under endoscopy with the use of navigation views (Figure 3); (IX) after adequate foraminoplasty, the bone-plasty and working-tube position was confirmed through fluoroscopy, the fluoroscopic images were basically consistent with the navigation (Figure 4); (X) the ipsilateral ligamentum flavum was removed, and the endoscope with the Iseepoint in the left flushing canal was inserted to confirm the location (Figure 5); (XI) the surgical decompression of neural root could be performed from the dorsal ligamentum to ventral herniation, until the compression of the neural root was relieved (Figure 6).

\section{Fluoroscopy group}

The procedure was performed as above using imagingguided fluoroscopy, as described by Xiong et al. (10). 

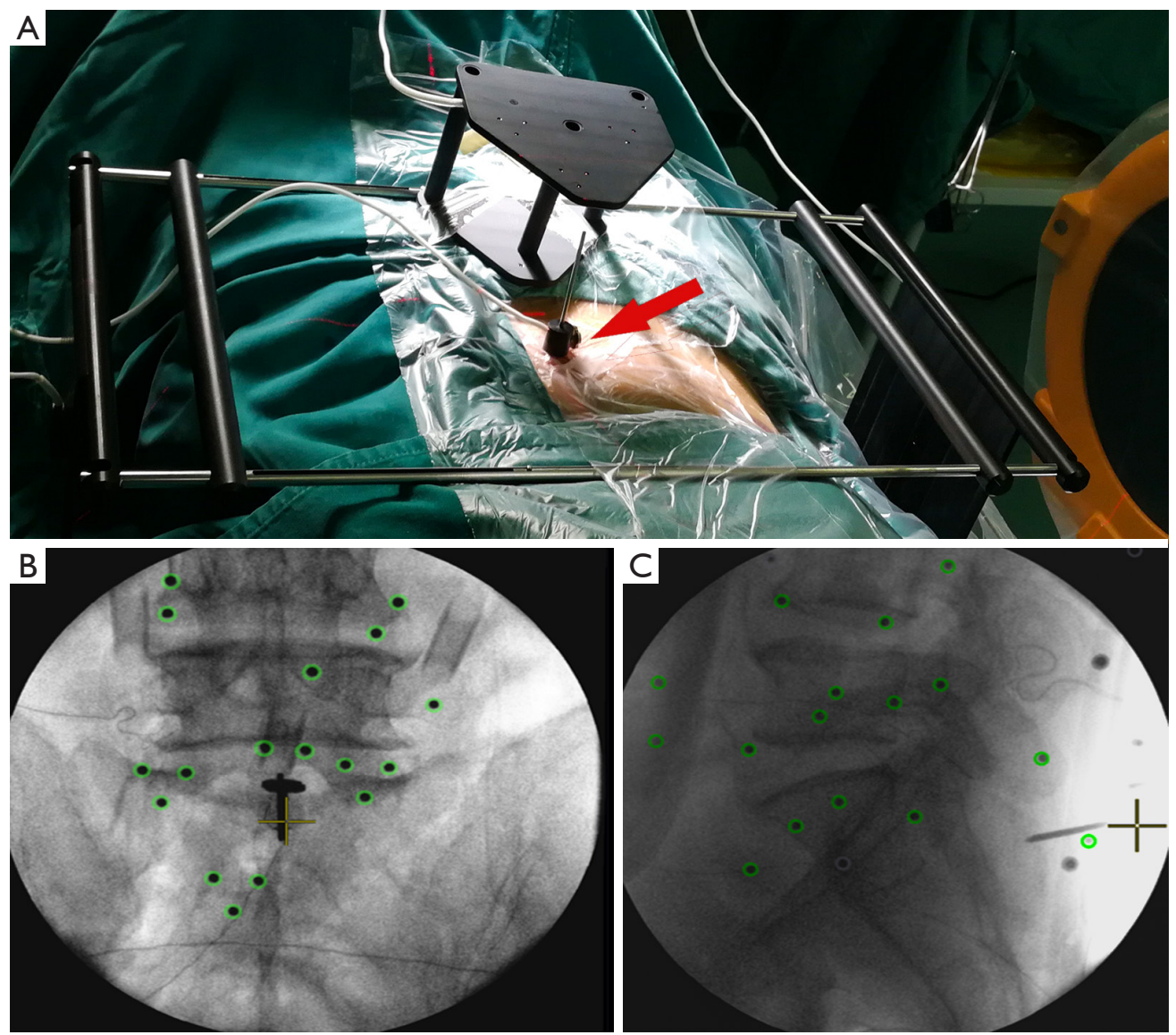

Figure 1 The patient tracker is attached to the spinous process (red arrow), and the mapper bridge is placed next to the localizer (A); the mapper bridge is identified by at least 17 markers on anteroposterior and lateral images (B,C).
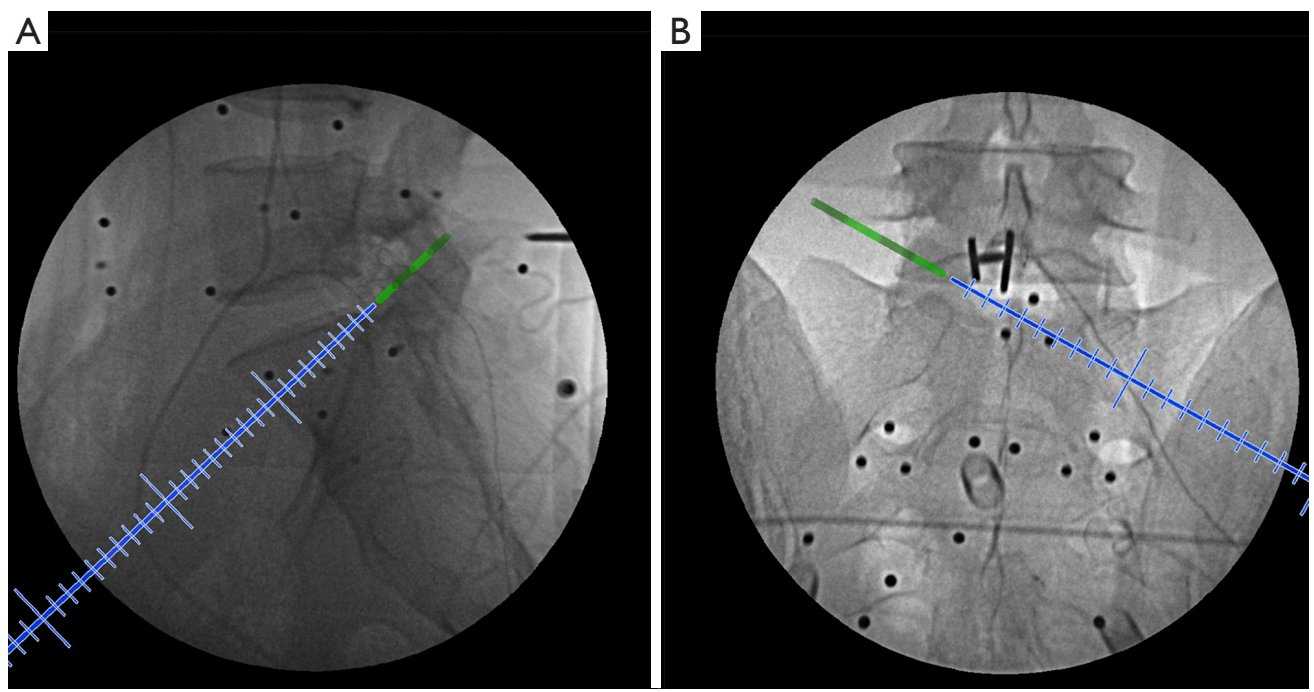

Figure 2 Real-time inline navigation trajectory help aim for the target point to superior articular process on lateral (A) and anteroposterior images (B). 

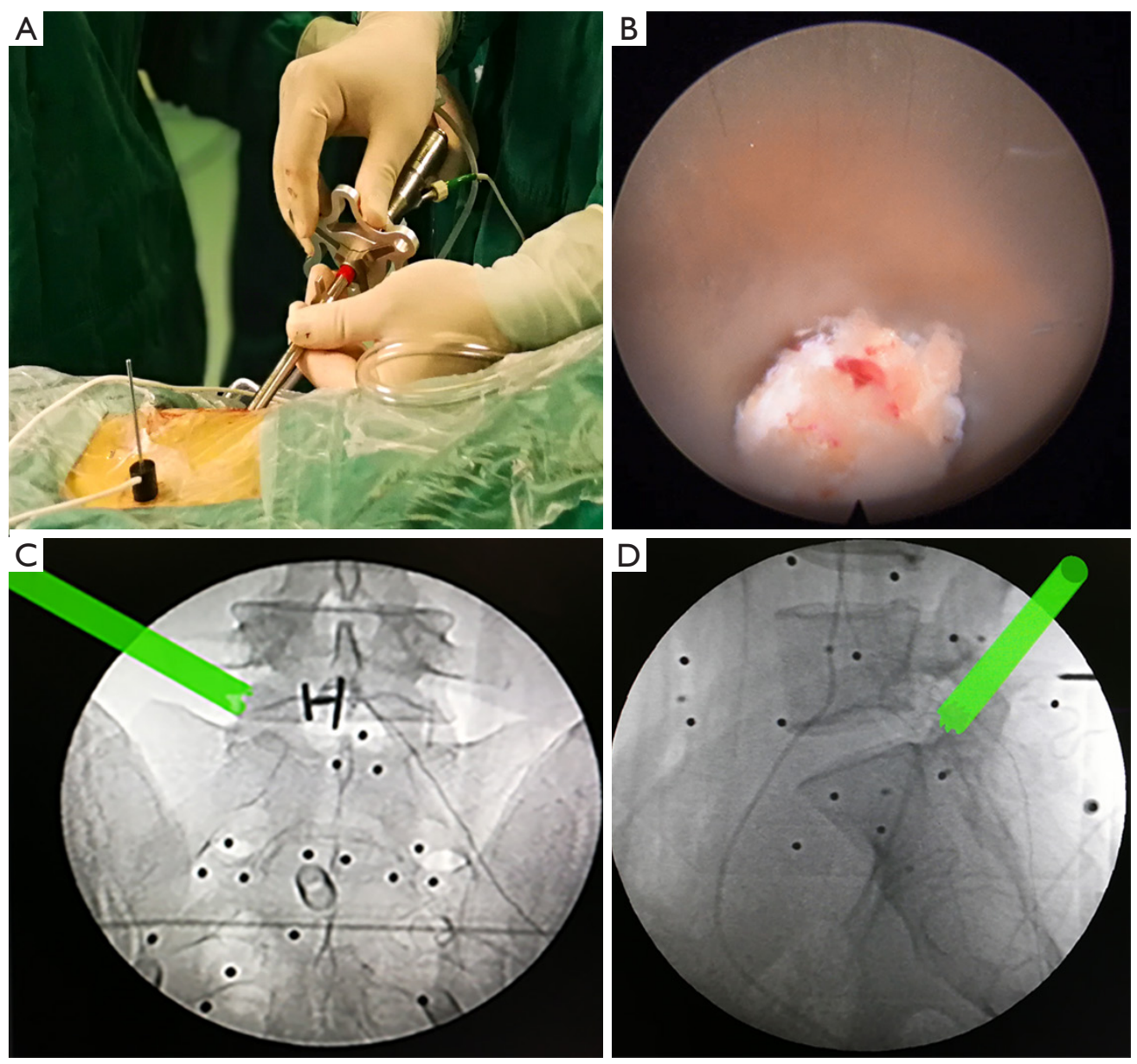

Figure 3 An appropriate reamer with handle is introduced along the half-serrated working cannula, then endoscope with the Iseepointer in the left flushing canal is inserted (A); the superior articular process is resected safely under the inline navigation and endoscopy views (B,C,D).

\section{Statistical analysis}

Statistical software PASS11 (NCSS, LLC, Kaysville, Utah, USA) was used to calculate the sample size of this study. Significance level (a) was defined 0.05 , and power calculations $(1-\beta)$ were defined 0.9 . The calculated minimum sample size was 35 patients in each group for this trial. Descriptive assessments and analytical statistics were performed with SPSS (version 21.0, SPSS, Chicago, IL, USA) depending on the group characteristics. Independent sample t tests, Chi-square tests, and Mann-Whitney U tests were conducted for baseline data, perioperative data, and Macnab outcome comparisons between the two groups. A nonparametric test was used for skewed variables. Repeatedmeasures analysis of variance was performed to evaluate the outcomes (VAS and ODI) at different follow-up points in the two groups. Paired $t$-tests were used to compare the scores at 1-, 3-, 6-, and 12-month follow-up with the baseline scores in each group. $\mathrm{P}<0.05$ (two sides) was considered significant, and a power analysis was conducted.

\section{Results}

The demographic characteristics of the two groups were not significantly different $(\mathrm{P}>0.05)$ (Table 1). Eighty-five patients were followed-up for at least 12 months.

The duration of surgery and cannula placement time were significantly shorter in the navigation group $(\mathrm{P}=0.03$, $\mathrm{P}<0.001)$. The radiation dose was significantly higher in the fluoroscopy group $(\mathrm{P}<0.001)$. There was significantly less intraoperative pain in the navigation group $(\mathrm{P}=0.038)$ (Table 2). VAS and ODI scores did not differ at baseline $(\mathrm{P}>0.05)$. VAS scores for back and leg pain improved significantly in both groups after surgery and improved 

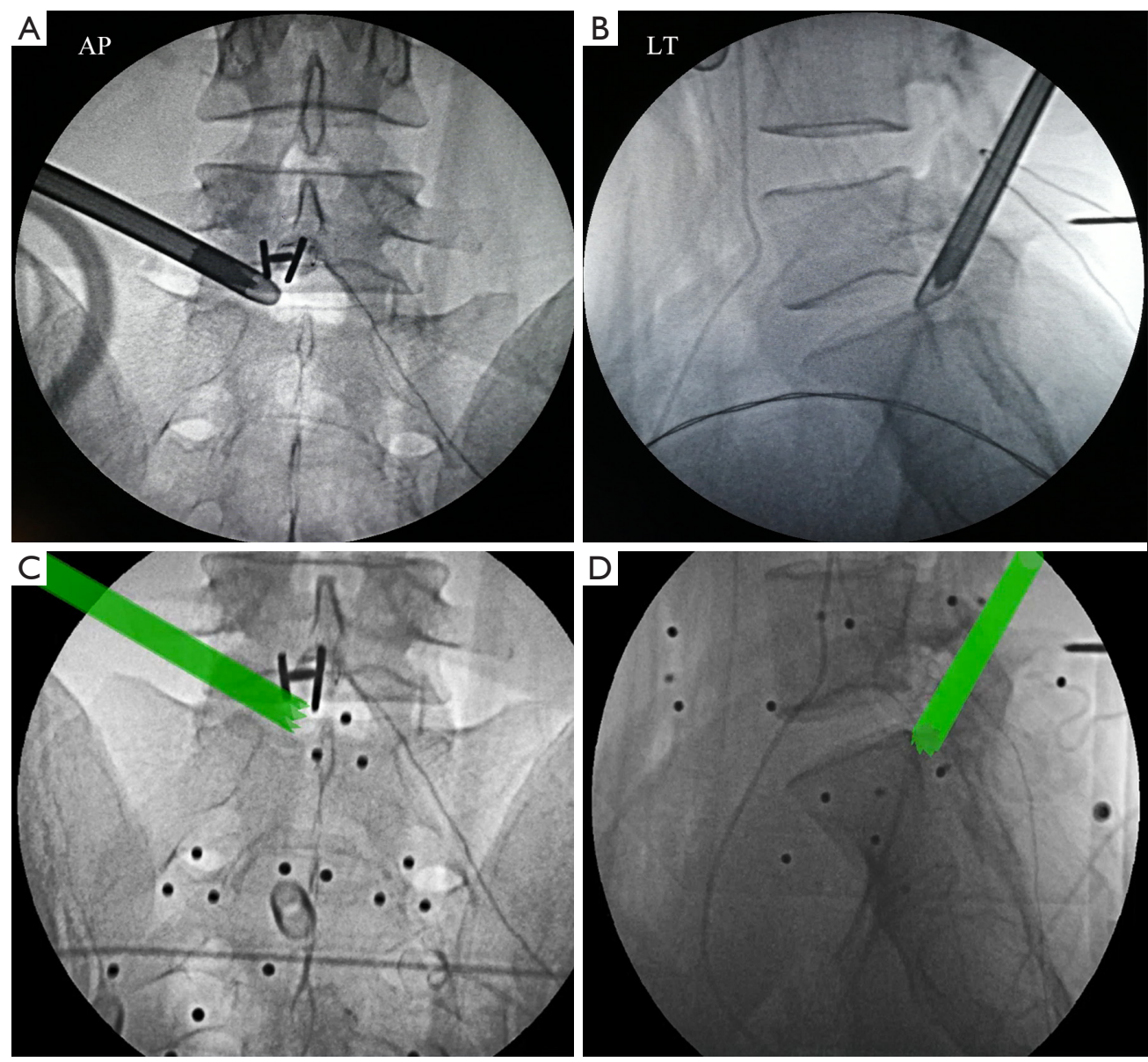

Figure 4 After adequate foraminoplasty, the bone-plasty and working-tube position was confirmed through fluoroscopy, the fluoroscopic images $(\mathrm{A}, \mathrm{B})$ were basically consistent with the navigation (C,D). AP, anteroposterior; LT, lateral.

gradually over time, as did the ODI scores. Repeatedmeasures analysis showed no significant differences in VAS and ODI scores between the two groups. The mean walking distance increased by $188 \mathrm{~m}$ (to $412 \mathrm{~m}$ ) and by $185 \mathrm{~m}$ (to $405 \mathrm{~m}$ ) in the navigation and fluoroscopy group, respectively (Table 3). According to the Macnab criteria, the total excellent and good rate was $90.6 \%$, the fair improvement rate was $7.1 \%$, and two patients had a poor result. Improvements in walking ability and Macnab criteria assessments at the 12-month follow-up did not differ between treatment groups (Table 4).

There were no serious complications. Transient numbness of the leg occurred in three patients and two patients complained of postoperative headache, and symptoms resolved with conservative management. One patient in group B had a poor result with incomplete decompression of the contralateral area, which was demonstrated by postoperative MRI and CT. Another patient in navigation group who had poor outcomes still reported 6 months of symptom relief after surgery. However, the patient complained of recurrence symptoms at the 12-month follow-up and then received minimally invasive transforaminal lumbar interbody fusion due to the recurrence of LSS.

\section{Discussion}

This prospective controlled study has demonstrated higher efficiency in foraminoplasty and lower levels of radiation exposure in the navigation group. In this article, we introduced a visualized endoscopic electromagnetic navigation system, consisting of a field generator, multiple field sensors, a processing computer, and matched instruments (10). The EMN procedure has high accuracy 

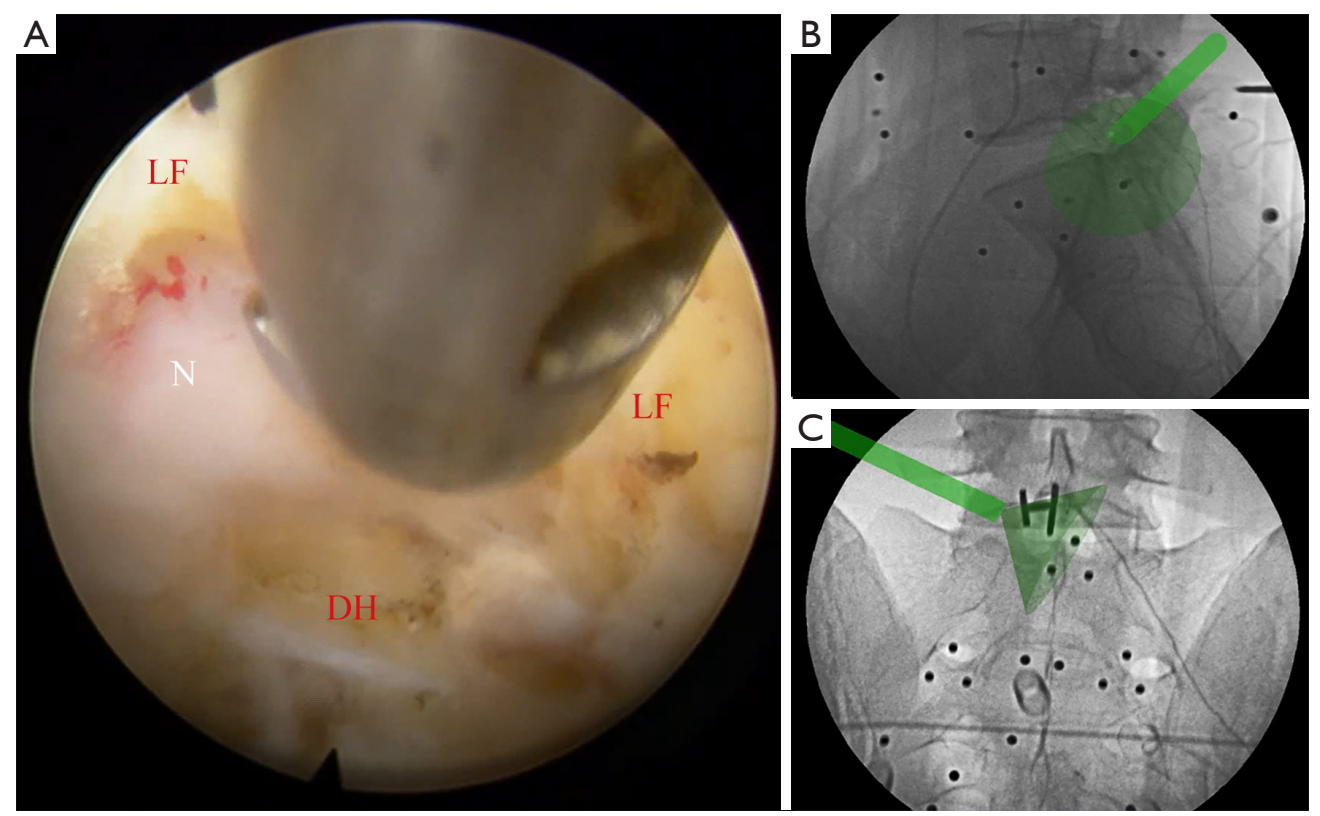

Figure 5 The ipsilateral ligamentum flavum is removed (A), and the endoscope with the Iseepoint in the left flushing canal is inserted to confirm the location (B,C). N, nerve; LF, ligamentum flavum; DH, disc herniation.
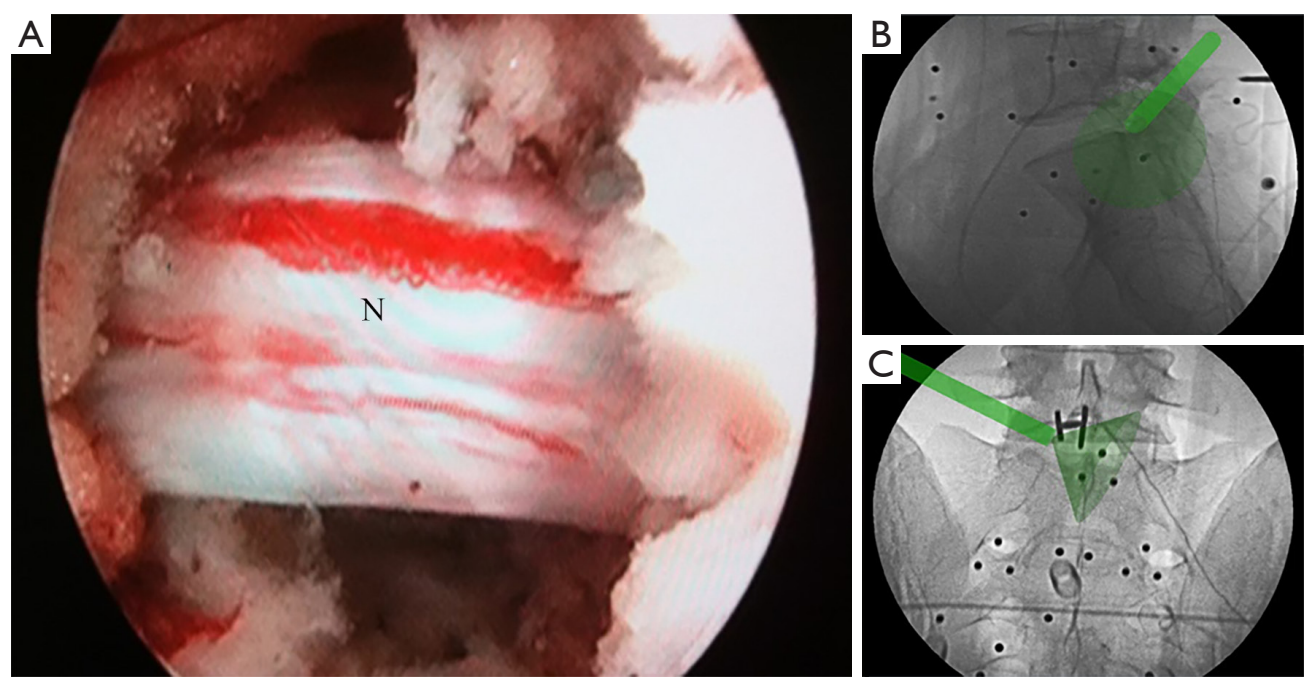

Figure 6 The surgical decompression of neural root can be performed from the dorsal to ventral, until the compression of the neural root is relieved (A); the real-time decompression location on lateral and anteroposterior images (B,C). N, nerve.

similar to optical navigation and eliminates the lineof-sight restrictions inherent in optical systems. The EMN procedure has the advantage of being compatible with nonrigid instruments, such as puncture needles, and the potential for expanded applications in MIS and percutaneous procedures, where the instruments to be tracked may be flexible $(21,22)$. EMN with an image- guided application of thoracic pedicle screws results in a decrease in fluoroscopic radiation exposure and the degree of perforation compared with conventional techniques using anatomic landmarks, however, problems can arise with field distortion when the transmitter is moved or when ferromagnetic substances approach the electromagnetic field (23). Under novel EMN guidance systems, surgeons 
Table 1 Demographic characteristic of the enrolled patients

\begin{tabular}{|c|c|c|c|}
\hline Characteristic & Group A & Group B & $P$ value \\
\hline Cases & 41 & 44 & \\
\hline Sex & & & 0.831 \\
\hline Male & 27 & 28 & \\
\hline Female & 14 & 16 & \\
\hline Age (year) & & & 0.993 \\
\hline Range & $19-73$ & $23-67$ & \\
\hline Mean \pm stand. Dev. & $47.39 \pm 14.76$ & $47.36 \pm 11.72$ & \\
\hline Levels involved & & & 0.627 \\
\hline L3-L4 & 3 & 4 & \\
\hline L4-L5 & 16 & 21 & \\
\hline L5-S1 & 22 & 19 & \\
\hline Type of stenosis & & & 0.387 \\
\hline Central stenosis & 11 & 8 & \\
\hline Lateral recess stenosis & 21 & 29 & \\
\hline Both & 9 & 7 & \\
\hline Severity of stenosis & & & 0.168 \\
\hline Schizas grade $\mathrm{C}$ & 24 & 32 & \\
\hline Schizas grade D & 17 & 12 & \\
\hline Body mass index & & & 0.751 \\
\hline$<18.5$ & 2 & 4 & \\
\hline $18.5-23.9$ & 18 & 19 & \\
\hline $24-27.9$ & 18 & 16 & \\
\hline$\geq 28$ & 3 & 5 & \\
\hline Follow-up & & & 0.324 \\
\hline Mean \pm stand. Dev. & $14.3 \pm 2.2$ & $14.8 \pm 2.2$ & \\
\hline
\end{tabular}

Group A, navigation group; Group B, fluoroscopy group.

can obtain a $3 \mathrm{D}$ anatomical structure of the spine or multiplanar imaging reconstruction, and surgical instruments can be tracked in real time for $3 \mathrm{D}$ space. The surgeon can synchronously obtain the local magnification operative view and the whole decompression site to confirm adequate decompression with high efficiency and low levels of radiation exposure.

LSS is the most common lumbar degenerative disease in elderly patients (24). LSS is characterized by narrowing of the spinal canal as a consequence of bone and soft tissue
Table 2 Perioperative data of all patients

\begin{tabular}{|c|c|c|c|}
\hline Variables & Group A & Group B & $P$ value \\
\hline Duration of surgery (min) & $74.5 \pm 20.5$ & $90.4 \pm 25.9$ & $0.03^{*}$ \\
\hline Cannula placement time (min) & $18.6 \pm 8.3$ & $35.3 \pm 15.1$ & $<0.001^{*}$ \\
\hline Radiation dose (mGy) & $3.4 \pm 1.0$ & $8.5 \pm 2.0$ & $<0.001^{*}$ \\
\hline Blood loss (mL) & $13.4 \pm 11.3$ & $13.6 \pm 10.3$ & 0.925 \\
\hline Intraoperative pain & & & $0.038^{*}$ \\
\hline Mild & 25 & 15 & \\
\hline Moderate & 13 & 21 & \\
\hline Severe & 3 & 8 & \\
\hline Intolerable & 0 & 0 & \\
\hline $\begin{array}{l}\text { Postoperative hospitalization } \\
\text { stay (days) }\end{array}$ & $2.3 \pm 0.8$ & $2.6 \pm 0.9$ & 0.190 \\
\hline
\end{tabular}

degeneration, including osteophyte formation, degeneration of the ligamentum flavum, disc herniation, and facet hypertrophy (1-4). As a result of the pathogenesis of LSS, both ventral decompression for disk herniation and dorsal nerve decompression for ligamentum flavum and facet hypertrophy should be performed $(25,26)$. Nevertheless, the degree of spinal instability is dependent on the extent of resection of the articular joints and ligamentous tissues. Two high-level randomized controlled trials revealed no clinical benefit 2 years after surgery by adding fusion surgery to decompression surgery $(5,6)$. Microscopic unilateral laminotomy with bilateral decompression via a unilateral approach has been used in generative lumbar stenosis with good postoperative outcomes reported (27). During the PTELD technique, dorsal decompression was achieved by partial resection of the ventral SAP and ligamentum flavum, but the structure of the facet joint capsule was still intact (10). The procedure can be performed with local anesthesia, especially in older patients who cannot tolerate conventional surgery under general anesthesia.

In our study, functional improvements were consistent with the previous literature (8). The excellent and good improvement rate in all patients was $90.6 \%$, and the VAS $(\mathrm{P}<0.001)$ and $\mathrm{ODI}(\mathrm{P}<0.001)$ scores improved significantly in both groups after surgery. We did not observe significant differences between the groups with respect to reductions in the VAS (Back, $\mathrm{P}=0.980 ; \mathrm{Leg}, \mathrm{P}=0.724)$ and $\mathrm{ODI}(\mathrm{P}=0.243)$ 
Table 3 Preoperative, follow-up VAS, ODI, scores and 6-minute walk assessment

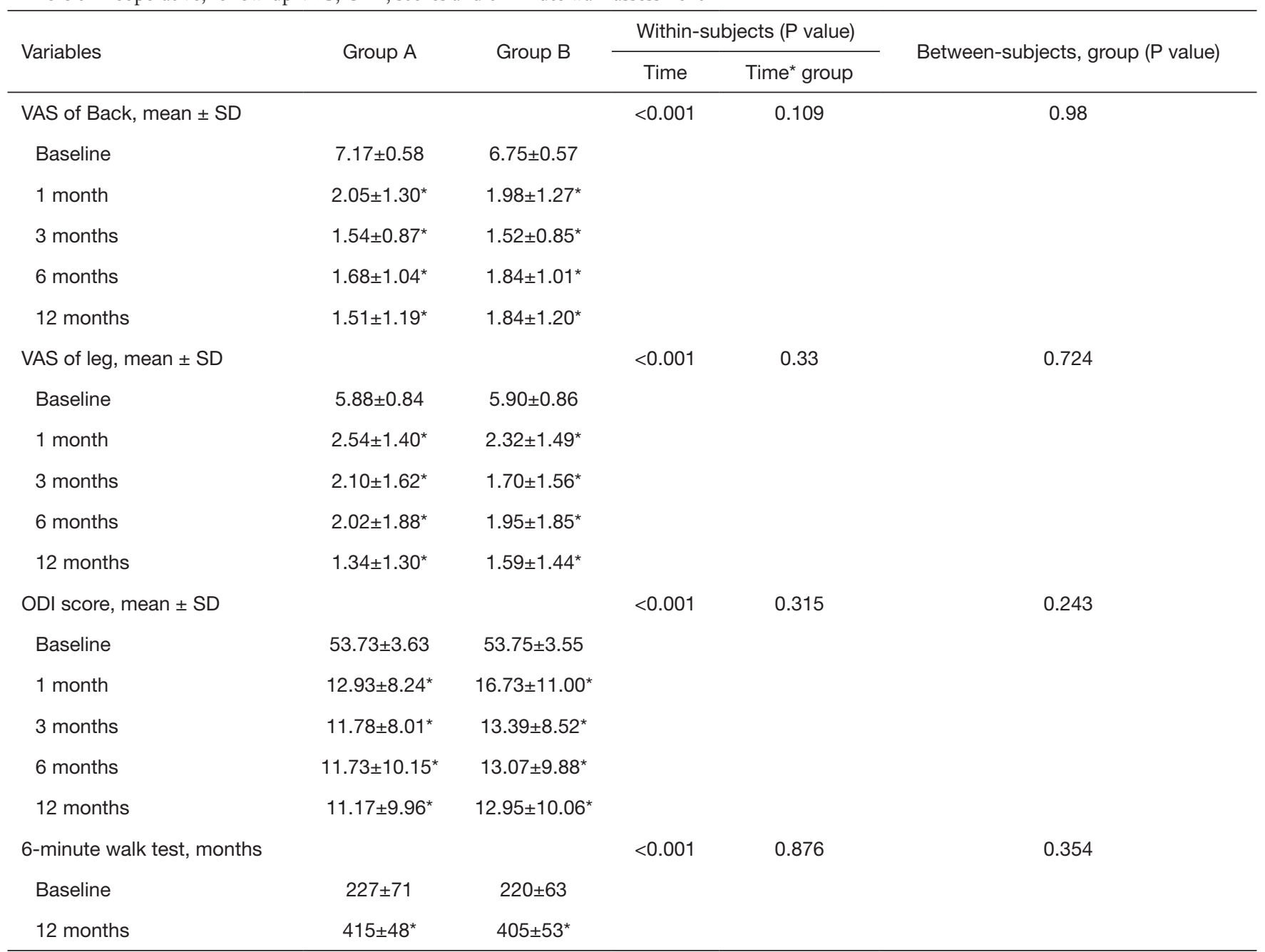

* $\mathrm{P}<0.05$ for significant improvement compared with baseline assessment. VAS, visual analog scale; ODI, Oswestry Disability Index; Group A, navigation group; Group B, fluoroscopy group.

Table 4 Modified Macnab criteria

\begin{tabular}{llcc}
\hline Outcome & Description & Group A & Group B \\
\hline Excellent & Complete relief of symptoms & 26 & 29 \\
Good & Marked improvement but occasional pain & 12 & 0.929 \\
Fair & Improved functional capacity and the need for pain medications & 2 & 4 \\
Poor & Unimproved symptoms or worsening & 1 & 1 \\
\hline
\end{tabular}

Group A, navigation group; Group B, fluoroscopy group.

scores. Improvements in walking ability $(\mathrm{P}=0.354)$ and Macnab criteria assessments $(\mathrm{P}=0.929)$ at the 12 -month follow-up with the use of subjective patient assessments did not differ between the treatment groups. The assessment of long-term clinical outcomes and randomized controlled trials should be considered to provide more evidence-based conclusions in the future.

During surgery, with the aid of real-time 3D navigation 
views, the entire puncture trajectory targeted at the tip of the SAP could be designed accurately, and the proper skin entry point was easily selected. Possible bony obstructions, including a high iliac crest and hypertrophic transverse processes, can be easily avoided with navigation views. In our study, the duration of surgery and cannula placement time were shorter, the radiation dose was lower, and there was less pain in the navigation group. Most of the severe pain during foraminoplasty was assumed to originate from exiting nerves and the posterior longitudinal ligament instead of the SAP. Under EMN guidance systems, surgeons can obtain a total $3 \mathrm{D}$ anatomical structure of the spine and can synchronously obtain a local magnification operative view. Surgical instruments can be tracked in real time for precise targeting of the puncture and foraminoplasty. Nevertheless, imaging-guided surgery is an adjunct technology to fluoroscopic imaging and is not intended to replace it. In contrast, the frequency of imaging will decrease and be limited to pivotal procedural checks for positioning, thus decreasing overall radiation exposure and enhancing safety and efficiency in foraminoplasty.

In terms of the current technical status, surgical indications for percutaneous transforaminal endoscopy are expanded from soft tissue pathologies to LSS. However, the learning curve remains flat, and most spinal surgeons remain unfamiliar with the technique. Surgical decompression of the dural sac or nerve roots starts from the dorsal bony-plasty to ventral decompression. Navigable endoscopic punches and curettes can help remove thickened ligaments and osteophytes delicately and efficiently, as demonstrated by our results. Patients with LSS combined with DH who complain of unilateral lower limb radiculopathy are considered appropriate for PTELD (11). To comply with the indication to obtain optimized clinical results, only patients with unilateral neurological intermittent claudication or radicular leg pain were included in our study, and some symptomatic elderly patients without herniation or sciatica symptoms were excluded. Moreover, there are some features of intraoperative fluoroscopy, such as magnification, distortion, and parallax. Therefore, the intraoperative C/G-Arm should be compatible with electromagnetic navigation and calibrated yearly.

The novel electromagnetic tracking surgical navigation system used in this investigation integrates intraoperative fluoroscopy with preoperative CT-based data to achieve intraoperative virtual $3 \mathrm{D}$ visualization in real time. Traditional optical navigation systems, which are mostly based on intraoperative cone-bone CT scans to obtain anatomical data, have been reported to increase radiation in scoliosis surgery (28). Unlike optical navigation systems, the EMN technique does not require an optical reference frame outside the site or on the instruments that can interfere with the normal workflow and eliminate line-ofsight restrictions. The primary limitation of the technology is the potential distortion of the electromagnetic field, so nonferromagnetic instruments and carbon fiber operating room tables should be prepared. Stainless steel objects should be kept more than $10 \mathrm{~cm}$ from electromagnetic computer surgery instruments to minimize electromagnetic interference (29).

\section{Conclusions}

In conclusion, compared to fluoroscopy, the novel EMN technique for PTELD in LSS patients can achieve similar clinical symptom improvement results. Furthermore, higher efficiency in foraminoplasty and lower radiation exposure levels were demonstrated. This system is easy to handle and does not limit the workflow. The electromagnetic tracking surgical navigation system can integrate intraoperative fluoroscopy with preoperative CT-based data to achieve intraoperative virtual 3D visualization in real time, which is a breakthrough in progress and will further enhance navigation ability and clinical application in more hospitals.

\section{Acknowledgments}

Funding: This work was supported by the technological innovation project of military clinical medicine of the second affiliated hospital of Army Medical University (Grant No. 2018JSLC0014) and Chongqing Science and Health Major Project Foundation (2019ZDXM018).

\section{Footnote}

Reporting Checklist: The authors have completed the CONSORT reporting checklist. Available at http://dx.doi. org/10.21037/atm-20-1877

Data Sharing Statement: Available at http://dx.doi. org/10.21037/atm-20-1877

Peer Review File: Available at http://dx.doi.org/10.21037/ atm-20-1877

Conflicts of Interest: All authors have completed the ICMJE 
uniform disclosure form (available at http://dx.doi. org/10.21037/atm-20-1877). The authors have no conflicts of interest to declare.

Ethical Statement: The authors are accountable for all aspects of the work in ensuring that questions related to the accuracy or integrity of any part of the work are appropriately investigated and resolved. The study was conducted in accordance with the Declaration of Helsinki (as revised in 2013). The procedure was approved by the Ethics Committee of Army Medical University and written informed consent was obtained from all patients. This random controlled clinical trial was registered in a public trial registry (ChiCTR1900028523).

Open Access Statement: This is an Open Access article distributed in accordance with the Creative Commons Attribution-NonCommercial-NoDerivs 4.0 International License (CC BY-NC-ND 4.0), which permits the noncommercial replication and distribution of the article with the strict proviso that no changes or edits are made and the original work is properly cited (including links to both the formal publication through the relevant DOI and the license). See: https://creativecommons.org/licenses/by-nc-nd/4.0/.

\section{References}

1. Katz JN, Harris MB. Clinical practice. Lumbar spinal stenosis. N Engl J Med 2008;358:818-25.

2. Weinstein JN, Tosteson TD, Lurie JD, et al. Surgical versus nonsurgical therapy for lumbar spinal stenosis. $\mathrm{N}$ Engl J Med 2008;358:794-810.

3. Kreiner DS, Shaffer WO, Baisden JL, et al. An evidencebased clinical guideline for the diagnosis and treatment of degenerative lumbar spinal stenosis (update). Spine J 2013;13:734-43.

4. Schizas C, Theumann N, Burn A, et al. Qualitative grading of severity of lumbar spinal stenosis based on the morphology of the dural sac on magnetic resonance images. Spine (Phila Pa 1976) 2010;35:1919-24.

5. Forsth P, Olafsson G, Carlsson T, et al. A Randomized, Controlled Trial of Fusion Surgery for Lumbar Spinal Stenosis. N Engl J Med 2016;374:1413-23.

6. Ghogawala Z, Dziura J, Butler WE, et al. Laminectomy plus Fusion versus Laminectomy Alone for Lumbar Spondylolisthesis. N Engl J Med 2016;374:1424-34.

7. Arai Y, Hirai T, Yoshii T, et al. A prospective comparative study of 2 minimally invasive decompression procedures for lumbar spinal canal stenosis: unilateral laminotomy for bilateral decompression (ULBD) versus muscle-preserving interlaminar decompression (MILD). Spine (Phila $\mathrm{Pa}$ 1976) 2014;39:332-40.

8. Phan K, Mobbs RJ. Minimally Invasive Versus Open Laminectomy for Lumbar Stenosis: A Systematic Review and Meta-Analysis. Spine (Phila Pa 1976) 2016;41:E91-e100.

9. den Boogert HF, Keers JC, Marinus Oterdoom DL, et al. Bilateral versus unilateral interlaminar approach for bilateral decompression in patients with singlelevel degenerative lumbar spinal stenosis: a multicenter retrospective study of 175 patients on postoperative pain, functional disability, and patient satisfaction. J Neurosurg Spine 2015;23:326-35.

10. Xiong C, Li T, Kang H, et al. Early outcomes of 270-degree spinal canal decompression by using TESSYSISEE technique in patients with lumbar spinal stenosis combined with disk herniation. Eur Spine J 2019;28:78-86.

11. Ahn Y. Percutaneous endoscopic decompression for lumbar spinal stenosis. Expert Rev Med Devices 2014;11:605-16.

12. Sairyo K, Chikawa T, Nagamachi A. State-of-the-art transforaminal percutaneous endoscopic lumbar surgery under local anesthesia: Discectomy, foraminoplasty, and ventral facetectomy. J Orthop Sci 2018;23:229-36.

13. Heo DH, Lee DC, Park CK. Comparative analysis of three types of minimally invasive decompressive surgery for lumbar central stenosis: biportal endoscopy, uniportal endoscopy, and microsurgery. Neurosurg Focus 2019;46:E9.

14. Wu J, Zhang C, Zheng W, et al. Analysis of the Characteristics and Clinical Outcomes of Percutaneous Endoscopic Lumbar Discectomy for Upper Lumbar Disc Herniation. World Neurosurg 2016;92:142-7.

15. Ao $\mathrm{S}, \mathrm{Wu} \mathrm{J}$, Zheng $W$, et al. A Novel Targeted Foraminoplasty Device Improves the Efficacy and Safety of Foraminoplasty in Percutaneous Endoscopic Lumbar Discectomy: Preliminary Clinical Application of 70 Cases. World Neurosurg 2018;115:e263-71.

16. Ao S, Wu J, Tang Y, et al. Percutaneous Endoscopic Lumbar Discectomy Assisted by O-Arm-Based Navigation Improves the Learning Curve. Biomed Res Int 2019;2019:6509409.

17. Schulz KF, Altman DG, Moher D, et al. CONSORT 2010 Statement: updated guidelines for reporting parallel group randomised trials. Trials 2010;11:32.

18. Macnab I. Negative disc exploration. An analysis of the 
causes of nerve-root involvement in sixty-eight patients. J Bone Joint Surg Am 1971;53:891-903.

19. Parker SL, Adogwa O, Paul AR, et al. Utility of minimum clinically important difference in assessing pain, disability, and health state after transforaminal lumbar interbody fusion for degenerative lumbar spondylolisthesis. J Neurosurg Spine 2011;14:598-604.

20. Steffen TM, Hacker TA, Mollinger L. Age- and genderrelated test performance in community-dwelling elderly people: Six-Minute Walk Test, Berg Balance Scale, Timed Up \& Go Test, and gait speeds. Phys Ther 2002;82:128-37.

21. Hahn P, Oezdemir S, Komp M, et al. Navigation of pedicle screws in the thoracic spine with a new electromagnetic navigation system: a human cadaver study. Biomed Res Int 2015;2015:183586.

22. Hahn P, Oezdemir S, Komp M, et al. A New Electromagnetic Navigation System for Pedicle Screws Placement: A Human Cadaver Study at the Lumbar Spine. PLoS One 2015;10:e0133708.

23. Sagi HC, Manos R, Benz R, Ordway NR, Connolly PJ. Electromagnetic field-based image-guided spine surgery part one: results of a cadaveric study evaluating

Cite this article as: $\mathrm{Wu} \mathrm{J}$, Ao S, Liu H, Wang W, Zheng W, Li C, Zhang C, Zhou Y. Novel electromagnetic-based navigation for percutaneous transforaminal endoscopic lumbar decompression in patients with lumbar spinal stenosis reduces radiation exposure and enhances surgical efficiency compared to fluoroscopy: a randomized controlled trial. Ann Transl Med 2020;8(19):1215. doi: 10.21037/atm-20-1877 lumbar pedicle screw placement. Spine (Phila Pa 1976) 2003;28:2013-8.

24. Shamji MF, Goldstein CL, Wang M, et al. Minimally Invasive Spinal Surgery in the Elderly: Does It Make Sense? Neurosurgery 2015;77 Suppl 4:S108-15.

25. Machado GC, Ferreira PH, Yoo RI, et al. Surgical options for lumbar spinal stenosis. Cochrane Database Syst Rev 2016;11:CD012421.

26. Issack PS, Cunningham ME, Pumberger M, et al. Degenerative lumbar spinal stenosis: evaluation and management. J Am Acad Orthop Surg 2012;20:527-35.

27. Armin SS, Holly LT, Khoo LT. Minimally invasive decompression for lumbar stenosis and disc herniation. Neurosurg Focus 2008;25:E11.

28. Urbanski $W$, Jurasz $W$, Wolanczyk $M$, et al. Increased Radiation but No Benefits in Pedicle Screw Accuracy With Navigation versus a Freehand Technique in Scoliosis Surgery. Clin Orthop Relat Res 2018;476:1020-7.

29. Stevens F, Conditt MA, Kulkarni N, et al. Minimizing electromagnetic interference from surgical instruments on electromagnetic surgical navigation. Clin Orthop Relat Res 2010;468:2244-50. 